\title{
AE Aquarii continuum emissions from Radio low frequencies to Infra-Red
}

\author{
Meil Abada-Simon \\ Observatoire de Paris-LESIA-CNRS-UMR8109, 92195 Meudon, France
}

\begin{abstract}
AE Aquarii radio flares are attributed to synchrotron optically thick emission from expanding plasma clouds, and the turnover frequency and the optically thin part of the synchrotron spectrum seem to be between the sub-millimetre and infra-red wavelengths. We present the various processes which may affect the synchrotron radiation at both very low (radio) frequencies and at higher energies, and what they infer on the properties of the radio flare source and its surroundings. We also envisage that both thermal and non-thermal emission processes may explain the puzzling spectrum of AE Aqr.
\end{abstract}

\section{Introduction}

\subsection{General properties of AE Aquarii}

AE Aquarii is an unusual Intermediate Polar which White Dwarf (WD) magnetic field strength is $10^{4} \leq B_{W D}(G) \leq 10^{7}$ (Lamb \& Patterson 1983; Lamb 1988; Stockman et al. 1992); it is the most asynchronous Cataclysmic Variable with a White Dwarf spin period of $33.08 \mathrm{~s}$ and an orbital period of $9.88 \mathrm{hr}$. Its binary separation is $a=1.8 \times 10^{9} \mathrm{~m}$. It is a "propeller", that is, no accretion disc is formed, and most of the accreting material is expelled from system (Wynn, King \&Horne 1997). AE Aqr is detected in all wavelength ranges, from $\mathrm{TeV}$ $\gamma$-rays to radio (Bowden et al. 1992, Meintjes et al. 1992, Meintjes et al. 1994, and other papers in these proceedings).

\subsection{Radio to Infrared emission(s)}

In radio, $\mathrm{AE}$ Aqr exhibits both quiescent emission and radio flares of various amplitudes on timescales from minutes to hours. It is almost always detected, except between some flares, and in the middle of a few flares (Abada-Simon et al. $2003 \mathrm{~b}$ ). Assuming a spherical source diameter not larger than the binary separation $\left(a \simeq 1.8 \times 10^{9} \mathrm{~m}\right)$, and that the emission is isotropic, the Rayleigh-Jeans law implies $T_{b} \geq 10^{10}-10^{12} \mathrm{~K}$ at $1-21 \mathrm{~cm}$ : this implies a non thermal emission. Since it is of wide bandwidth $(\Delta \nu / \nu \geq 2$, Abada-Simon et al. 1995a) and non polarised (polarisation degree $<10 \%$ ), it is attributed to an incoherent emission process. Since the spectral flux density $S_{\nu}$ increases with frequency $\nu$ as $S_{\nu} \propto \nu^{\alpha}$, where the spectral index is $\alpha \simeq+0.5$, Bastian, Dulk \& Chanmugam (1988) explained the radio flares as the superposition of synchrotron optically thick emission from 
a power-law energy distribution of $\sim 1-15 \mathrm{MeV}$ electrons in spherical clouds in adiabatic expansion. Another model has been recently suggested to explain the average observed spectrum from radio to far infrared (Venter \& Meintjes 2003, and Meintjes \& Venter 2003). Since AE Aqr is almost always emitting down to $21 \mathrm{~cm}$, the plasma frequency is $\nu_{p}(H z) \simeq 9000 \sqrt{n_{e}}<1.4 \times 10^{9} \mathrm{~Hz}$, which implies that the electron number density is $n_{e}<2.4 \times 10^{10} \mathrm{~cm}^{-3}$.

AE Aqr radio source was once resolved by Niell in 1988 (private communication) with VLBI at $3.6 \mathrm{~cm}$, leading to a radio source diameter changing from $\leq 1.6 \mathrm{a}$ (unresolved) to $4 \mathrm{a}$ (resolved) in $30 \mathrm{~min}$, which is consistent with the above model. However, a much smaller radio source size has been estimated: dips at both 2 and $3.6 \mathrm{~cm}$ were attributed most likely to eclipses of the radio source through free-free absorption by gas blobs accreting from the secondary star (Abada-Simon et al. 1995b); the inferred size may be down to $10^{7} \mathrm{~m}$ (of the order of the White Dwarf size), which implies $T_{b} \geq 10^{12} \mathrm{~K}$ : apart from synchrotron emission from higher energy $(\sim 100 \mathrm{MeV})$ electrons, this also suggested the possible presence of coherent emission in the radio domain (Abada-Simon et al. 1996).

The electrons accreting from the secondary are thought to be trapped in the White Dwarf magnetosphere and accelerated by magnetic pumping, producing permanently synchrotron emission, until a cloud is expelled, thus producing a flare (Kuijpers et al. 1997). However, no (quasi-)periodicity was found at 3.6 $\mathrm{cm}$, so that a source very close to the White Dwarf remains uncertain (Bastian, Beasley \& Bookbinder 1996).

Finally, at both $0.8 \mathrm{~mm}$ and $90 \mu \mathrm{m}$, the flux density is found to be $\simeq 110$ mJy, which is also the value of the $3 \sigma$ upper limit at $170 \mu \mathrm{m}$ (Abada-Simon et al. 2003a). Assuming again an isotropic emission from a spherical source diameter $\leq a$, we find $T_{b} \geq 10^{7} \mathrm{~K}$ at $0.8 \mathrm{~mm}$ and $T_{b} \geq 10^{6} \mathrm{~K}$ at $90 \mu \mathrm{m}$ : these results suggest that the far infrared emission may be of thermal origin, and most probably that gyro-synchrotron $(1<\gamma \leq 2-3)$ emission from a thermal or nonthermal electron energy distribution is present, since it leads to $T_{b} \simeq 10^{7}-10^{9} \mathrm{~K}$.

Clearly, many uncertainties remain on the radio to infrared emission(s) of AE Aqr: what are the initial and final values of the source parameters (size, location, density, magnetic field strength, energy distribution(s)...), how do they evolve with time, what are the emission and the acceleration process(es), etc.? In Section 2, we compare the theoretical constraints concerning the synchrotron radiation (i.e. from a power-law energy distribution of electrons with $\gamma>3$ ) with the observational data, which are average spectral flux density values over about 15 years; in Section 3, we briefly mention alternatives to the non-thermal synchrotron emission, which are developed in Abada-Simon et al. 2003a). 


\section{Synchrotron radiation}

It is known that, if the electron energy distribution function is a power-law $N(E) \propto E^{-p}$ (spectral index $-p$ ), the synchrotron optically thin emitted flux density is also a power-law, of spectral index $-(p-1) / 2$, which implies a flux increasing with decreasing frequencies. The $90 \mu \mathrm{m}$ detection together with an IRAS upper limit at $60 \mu \mathrm{m}$ suggest a far Infra-Red optically thin synchrotron spectrum of spectral index $\leq-1.1$, implying $p \geq 3.2$. However, at both low frequencies and high energies, several processes affect the (predicted) synchrotron optically thin spectrum (e.g. Rybicki \& Lightman 1979, Melrose 1994).

\subsection{Attenuations of synchrotron radiation at low frequencies}

Synchrotron self-absorption Self-absorption of the synchrotron radiation leads to an optically thick spectrum in the form of $\nu^{5 / 2}$, where $\nu$ is the frequency. Assuming that the source is a sphere of angular diameter $\theta$ (in arcseconds), the turnover frequency, i.e. where the optical thickness is $\tau \approx 1$, is:

$\nu_{m}(\mathrm{~Hz})=3.4 \times 10^{7}\left(\frac{S_{\nu_{m}}}{\theta^{2}}\right)^{2 / 5} B^{1 / 5}$

where $S_{\nu_{m}}$ is the maximum flux density (in Jansky) measured at $\nu_{m}$, and $B$ is the magnetic field strength in the source (in Gauss in all this text). As $\nu_{m}$ depends little on B, measuring $S_{\nu_{m}}$ at $\nu_{m}$ leads to the source size.

On the other hand, assuming that the source magnetic field strength cannot exceed the lower limit of the White Dwarf magnetic field $\left(B<10^{4} \mathrm{G}\right)$ implies a small source size of $4 \times 10^{8} \mathrm{~m}$ for $\nu_{m}=400 \mathrm{GHz}(0.8 \mathrm{~mm})$ and $3 \times 10^{7} \mathrm{~m}$ for $\nu_{m}=3333 \mathrm{GHz}(90 \mu \mathrm{m})$; this latter value, which is as small as that inferred independently from eclipses at 2 and $3.6 \mathrm{~cm}$, confirms the possibility of $T_{b} \simeq 10^{12} \mathrm{~K}$, therefore of synchrotron from $100 \mathrm{MeV}$ electrons (Inverse Compton scattering by relativistic electrons limits, for synchrotron sources, $T_{b}$ to $\leq 10^{12} \mathrm{~K}$; Melrose 1994); for $B_{W D}$ up to $10^{7} \mathrm{G}$, the inferred source size is only 4 times larger. Also, both $\nu_{m}$ and $S_{\nu_{m}}$ decrease with time, which leads to two conclusions: it may explain that ISO detected AE Aqr firstly at $90 \mu \mathrm{m}$, but not immediately after at $170 \mu \mathrm{m}$; a delay of low-frequency flare peaks with respect to higher frequency peaks should be measured.

Finally, the observed average radio spectrum (from $21 \mathrm{~cm}$ to $0.8 \mathrm{~mm}$, average spectral index $\simeq+0.5$ ) is less steep than the theoretical, self-absorbed instantaneous synchrotron spectrum of a single power-law energy distribution (spectral index $\simeq+2.5$ ).

Several reasons are invoked to explain why the optically thick spectrum can be either steeper than the self-absorbed one, or less steep, up to flat. The source may be non-spherical, inhomogeneous and/or composed of several electron energy distribution functions. Let us now consider two processes leading to spectra steeper than both the theoretical self-absorbed synchrotron and AE Aqr observed average radio spectrum (see Melrose 1994 for detailed explanations).

Optically thick spectra steeper than self-absorbed synchrotron Free-free absorption by an external plasma of thermal electrons at temperature $\mathrm{T}$ and located 
on the line of sight (e.g. wind), which involves $\tau_{f f} \propto \nu^{-2}$, implies a very sharp $\left(\exp ^{-\tau_{f f}}\right)$ cut off at frequencies below the absorption frequency:

$\nu_{a}(M H z)=n_{e} \sqrt{L} / 2$

where $L$ is the size of the plasma (in pc). This phenomenon was invoked earlier to explain the few radio dips observed at $\nu_{a} \simeq 8.4 \& 15 \mathrm{GHz}$, and since the absorbing blob transverse size was estimated to be $6 \times 10^{6}-5 \times 10^{8} \mathrm{~m}$ (AbadaSimon et al. 2003b), the external plasma density is $n_{e} \geq(1-20) \times 10^{8} \mathrm{~cm}^{-3}$; the lack of simultaneous data prevents from confirming absorption at lower frequencies.

The Razin effect (or suppression), which is not an absorption effect, reduces considerably the synchrotron emission when a thermalised plasma is present in the emitting region, so that the medium refraction index becomes significantly $<1$. It occurs at frequencies below the Razin-Tsytovitch frequency:

$\nu_{R T}(M H z)=\frac{2 \nu_{p}^{2}}{3 \nu_{o}} \simeq 2 \times 10^{-5} n_{e} / B_{\perp}$ where $\nu_{o}(M H z) \simeq 2.8 B$ is the cyclotron frequency, and $B_{\perp}$ is the magnetic field component which is perpendicular to the electron velocity (e.g. Ramaty et al. 1974). The detection of AE Aqr down to $21 \mathrm{~cm}$ implies $\nu_{R T}<1400 \mathrm{MHz}$, and, assuming again that $B($ source $)<10^{4} \mathrm{G}$, the inferred plasma density is $n_{e}<7 \times 10^{11} \mathrm{~cm}^{-3}$.

On the other hand, three processes lead to spectra less steep than the selfabsorbed synchrotron.

Optically thick spectra less steep than self-absorbed synchrotron If the synchrotron emitting plasma is uniformly mixed with the thermal plasma, freefree absorption by an internal plasma results from the optically thin emission $\propto \nu^{-(p-1) 72}$ being balanced by free-free absorption $\propto \nu^{-2}$ : the flux density below $\nu_{a}$ has a spectral index of $(5-p) / 2$. The spectral index observed for AE Aqr average radio spectrum is 0.5 , which would imply $p=4$. This thermal absorption would dominate over Razin suppression only if $\nu_{R T}<\nu_{a}$, which would lead to $B_{\perp}>4 \times 10^{-5} / \sqrt{L}$; with $B_{\perp}<10^{4} \mathrm{G}$, we find $L>2.7 \times 10^{6} a$ : this is much too large, which suggests that it is Razin suppression which dominates over absorption by an internal thermalised plasma.

Induced Compton scattering, which is important when the Thomson scattering's optical depth is large, leads to a spectral index of $(5-p) / 4$, which would imply $p=3$ for AE Aqr.

Finally, below the critical frequency corresponding to the electron minimum energy, $\nu_{c r}\left(E_{\min }\right)$, the spectrum varies as $\nu^{1 / 3}$. This spectrum has never been observed in any astrophysical source, probably because the minimum energy to accelerate particles is very low, so that: $\nu_{c r}\left(E_{\min }\right)<1.4 \mathrm{GHz}$ for AE Aqr plasma clouds. 


\subsection{Energy losses of relativistic electrons}

The losses by synchrotron radiation steepen the power-law electrons energy distribution, giving:

$N(E) \propto E^{-(p+1)}$ at frequencies $>\nu_{k}(H z)=3.4 \times 10^{8} B_{\perp}^{-3} t_{s y n}^{-2}$

where $t_{\text {syn }}$ is the synchrotron loss time (or the age of the source), in years, obtained from $t_{s y n}(y r) \propto-1 /\left(\frac{d E}{E d t}\right) \propto B_{\perp}^{-2} E_{o}^{-1}$; the optically thin spectrum thus steepens, changing its spectral index from $-(p-1) / 2$ to $-(2 p+1) / 3 . \nu_{k}$ decreases with time and is probably in the infrared.

If the steepening of the synchrotron optically thin spectrum occurs at $\nu_{k} \geq c /(90 \mu m)$, then the spectral index estimated from observations (-1.1) leads to $p \geq 1.15$, and, for $B=1,10,100,1000$ and $10^{4} \mathrm{G}$, we find respectively $t_{\text {syn }} \simeq 3.65$ days, $2.8 \mathrm{hr}, 5 \mathrm{~min}, 10 \mathrm{~s}$ and $0.3 \mathrm{~s}:$ if the initial magnetic field in the source is weak $(<10 \mathrm{G})$, then the time for a steepening of the optically thin synchrotron spectrum is larger than the timescale of the flares (minutes to hours in radio), that is, too long to happen; but if $B>10 \mathrm{G}$, then the steepening may be observed before the source has released all its energy. Again, the evolution with time of plasmoids instantaneous spectra is necessary to constrain their parameters.

In addition to synchrotron losses, losses by inverse Compton scattering may also come into play: collisions of low energy photons (radio to optical) with relativistic electrons transfer energy to photons (UV to $\gamma$ ). They also make the higher energy electrons loose more energy. $U$ being the energy density of the radiation field, for $\nu>\nu_{q}(G H z) \simeq 4 B_{\perp} /(U t)^{2}$, with $U$ in erg.cm ${ }^{-3}$, the ratio of loss times is $t_{\text {inv.Compt. }} / t_{\text {syn }} \propto B^{2} / U$ : if the magnetic energy density is $<U$, then the inverse Compton losses dominate over synchrotron losses.

Finally, losses by adiabatic expansion of a relativistic plasma are important if $t_{\text {adia.expans. }}$ is lower than the time spent by the particle in the region, i.e. for a plasma cloud expanding at the speed $v$ : $t_{\text {adia.expans. }}=r / v$ where $r$ is the source size. At high energies, synchrotron losses dominate over adiabatic expansion losses if $t_{\text {adia.expans. }}>t_{\text {syn }}$; for a far infrared source of $r \simeq 0.15 a$ expanding at $v=0.01 c$ (like the radio source once measured by VLBI), this condition is fulfilled if $B>300 G$. At radio frequencies, the reverse situation is often assumed.

\subsection{Polarisation of the synchrotron radiation}

In the optically thin case, if $B$ is uniform, for $p>1 / 3$, the degree of linear polarisation is: $\Pi_{t h i n}=\frac{p+1}{p+7 / 3}$. In the optically thick case, it is $\Pi_{t h i c k}=\frac{3}{6 p+13}$. Whereas $\Pi_{t h i n}$ is high for typical values of $p, \Pi_{t h i c k}$ is rather low: the expansion of the synchrotron emitting plasma cloud may be accompanied by a rotation of the electric vector.

The estimates made in the former sections suggest that $1.15 \leq p \leq 4$. The degree of linear polarisation in the optically thick case should thus be $8-15 \%$, but no significant value could be measured in the data between 1 and $21 \mathrm{~cm}$ (degree $<10 \%$ ): either the instrument was not sensitive enough to linear polarisation, or 
the synchrotron radiation travelled through an inhomogeneous medium, leading to a recorded degree of polarisation much lower than predicted by theory. In the optically thin case, the degree should be $62-79 \%$, but, to date, no polarisation degree could be measured below $1 \mathrm{~cm}$.

\section{Thermal and non-thermal emission processes?}

In the case of thermal bremsstrahlung, that is, free-free radiation from a thermal electron energy distribution, the emitting region is optically thick at low frequencies, so that the expected flux density varies as $\nu^{2}$ below a critical frequency $\nu_{c}$, and as $\nu^{-0.1}$ between $\nu_{c}$, and $k T / h$; above this latter high frequency, the optically thin spectrum then varies as $\exp ^{-h \nu /(k T)}$. In order to account for the far infrared measurement with thermal bremsstrahlung, a very large thermal plasma source of temperature $\simeq 575 \mathrm{~K}$ would be required (Abada-Simon et al. 2003a).

If the density is high enough, or if the temperature or the field strength is low enough, this process, which varies as $n^{2} T^{-1 / 2}$, dominates over gyro-synchrotron, which varies as $n T^{a} B^{b}$, with $a>1$ and $b>1$ (Dulk 1985). Gyro-synchrotron $(1<\gamma \leq 2-3)$ emission from a thermal or non-thermal electron energy distribution can account for the measured emissions from the far infrared to the radio if we assume that the far infrared source is small $(\sim 0.1 a)$ and that it expands to large values $(\geq 5 a)$ which are measurewd in radio. The spectrum of gyro-synchrotron emission from a thermal electron energy distribution varies as $\nu^{2}$ at low frequencies and as $\nu^{-8}$ at higher frequencies (Dulk 1985). For a non-thermal, power-law distribution, the expected spectrum varies as $\nu^{2.9}$ at low frequencies and as a power-law of index $-(2.7 \delta-3.6) / 3$ at higher frequencies (from Dulk 1985). In order to determine which emission processes dominate in radio and in infrared, a more complete spectrum of AE Aqr is necessary.

\section{Conclusion}

The whole picture to explain AE Aqr radio and far infrared emissions is not simply synchrotron radiation from a single power-law electron energy distribution, but rather emission processes from several distributions, including possibly a thermal distribution which may contribute to some of the measured emission. In order to modelise quantitatively the observed emission(s), one needs high sensitivity, multi-frequency, simultaneous data with high resolutions in both space, frequency, and time, and with the 4 Stokes parameters. In particular, the expected correlation between radio and high energies requires to be investigated.

Acknowledgments. M.A.S. is very grateful to M. Mouchet for her comments. 


\section{References}

Abada-Simon, M., Bastian, T.S., Bookbinder, J.A., Aubier, M., Bromage, G., Dulk, G.A., \& Lecacheux, A. 1995a, in : "Flares and Flashes", Proc. of the IAU Coll. 151, ed. J. Greiner, H.W. Duerbeck, \& R.E. Gershberg, LNP, Vol. 454, 268

Abada-Simon, M., Bastian, T.S., Horne, K., Robinson, E.L., \& Bookbinder, J.A. 1995b, in Proc. Cape Workshop on Magnetic Cataclysmic Variables, ed. D.A.H. Buckley \& B. Warner, ASP Conf. Series, Vol. 85, San Francisco, 355

Abada-Simon M., Bastian T.S., Fletcher L. et al. 1996, in "Radio emission from the stars and the Sun", eds. A.R. Taylor \& J.M. Paredes, ASP Conf. Series, 93, 182

Abada-Simon, M., Mouchet, M., Casares, J, (+ 13 co-authors) 2002, in "SF2A2002 Semaine de l'Astrophysique Française", eds. F. Combes \& D. Barret, EdP-Sciences (Editions de Physique), Conference Series, 497

Abada-Simon et al. 2003a, A\&A, submitted, in revision

Abada-Simon et al. 2003b, in preparation

Bastian, T.S., Dulk, G.A. \& Chanmugam, G. 1988, ApJ, 324, 431

Bastian, T.S., Beasley, T. \& Bookbinder, J.A. 1996, ApJ, 461, 1016

Bowden, C.C.G., Bradbury, S.M., Chadwick, P.M. (+ 8 co-authors) 1992, Astroparticule Physics, 1, 47

Dulk, G.A. 1985, Ann. Rev. Astron. Astrophys., 23, 169

Kuijpers, J., Fletcher, L., Abada-Simon, M., Horne K.D., Raadu M.A., Ramsay G. \& Steeghs D. 1997, A\&A, 322, 242

Lamb, D.Q. 1988, in "Polarised Radiation of Circumstellar Origin", Vatican City State/Tucson, AZ, Vatican Observatory/University of Arizona Press, 1988, 151

Lamb, D.Q. \& Patterson, J. 1983, in "Cataclysmic variables and related objects", Proc. of 72nd Colloquium, Dordrecht, D. Reidel Publishing Co., 1983,229

Meintjes, P.J., Raubenheimer, B.C., de Jager, O.C., Brink, C., Nel, H.I., North, A.R., van Urk, G. \& Visser, B. 1992, ApJ, 401, 325

Meintjes, P.J., de Jager, O.C., Raubenheimer, B.C., Nel, H.I., North, A.R., Buckley, D.A.H. \& Koen, C. 1994, ApJ, 434, 292

Meintjes, P. \& Venter, L. 2003, MNRAS, 341, 891

Melrose, D. 1994, in "Plasma Astrophysics", Lecture Notes of the Swiss Society for Astronomy and Astrophysics (SSAA), ed. A.O. Benz and T.J.L. Courvoisier, Springer, 113

Ramaty, R., Cheng, C.C. \& Tsuruta, S. 1974, ApJ, 187, 61

Rybicki, G.B. \& Lightman, A.P. 1979, Radiative Processes in Astrophysics, New York, Wiley-Interscience

Stockman, H.S., Schmidt, G.D., Berriman, G., Liebert, J., Moore, R.L. \& Wickramasinghe, D.T. 1992, ApJ, 401, 628

Venter, L. \& Meintjes, P.J. 2003, this proceedings 